\title{
Quantitative measurement of HER2 expression in breast cancers: comparison with 'real-world' routine HER2 testing in a multicenter Collaborative Biomarker Study and correlation with overall survival
}

Denise A Yardley ${ }^{1,2^{*}}$, Peter A Kaufman ${ }^{3}$, Weidong Huang ${ }^{4}$, Lea Krekow ${ }^{5}$, Michael Savin ${ }^{6}$, William E Lawler ${ }^{7}$, Stephen Zrada ${ }^{8}$, Alexander Starr ${ }^{9}$, Harvey Einhorn ${ }^{10}$, Lee S Schwartzberg ${ }^{11}$, John W Adams ${ }^{12}$, Yolanda Lie ${ }^{4}$, Agnes C Paquet ${ }^{4,15}$, Jeff Sperinde ${ }^{4}$, Mojgan Haddad ${ }^{4,16}$, Steve Anderson ${ }^{4}$, Marlon Brigino ${ }^{13}$, Rick Pesano ${ }^{4,17}$, Michael P Bates ${ }^{4,18}$, Jodi Weidler ${ }^{4,18}$ and Linda Bosserman ${ }^{14,19}$

\begin{abstract}
Introduction: Accurate assessment of HER2 status is critical in determining appropriate therapy for breast cancer patients but the best HER2 testing methodology has yet to be defined. In this study, we compared quantitative HER2 expression by the HERmark ${ }^{\text {TM }}$ Breast Cancer Assay (HERmark) with routine HER2 testing by immunohistochemistry (IHC) and fluorescence in situ hybridization (FISH), and correlated HER2 results with overall survival (OS) of breast cancer patients in a multicenter Collaborative Biomarker Study (CBS).

Methods: Two hundred and thirty-two formalin-fixed, paraffin-embedded breast cancer tissues and local laboratory HER2 testing results were provided by 11 CBS sites. HERmark assay and central laboratory HER2 IHC retesting were retrospectively performed in a blinded fashion. HER2 results by all testing methods were obtained in 192 cases.

Results: HERmark yielded a continuum of total HER2 expression ( $\mathrm{H} 2 \mathrm{~T}$ ) ranging from 0.3 to $403 \mathrm{RF} / \mathrm{mm}^{2}$ (approximately 3 logs). The distribution of H2T levels correlated significantly $(P<0.0001)$ with all routine HER2 testing results. The concordance of positive and negative values (equivocal cases excluded) between HERmark and routine HER2 testing was $84 \%$ for local IHC, 96\% for central IHC, 85\% for local FISH, and 84\% for local HER2 status. OS analysis revealed a significant correlation of shorter OS with HER2 positivity by local IHC (HR=2.6, $P=0.016)$, central IHC (HR=3.2, $P=0.015)$, and HERmark $(H R=5.1, P<0.0001)$ in this cohort of patients most of whom received no HER2-targeted therapy. The OS curve of discordant low (HER2 positive but H2T low, 10\% of all cases) was aligned with concordant negative (HER2 negative and $\mathrm{H} 2 \mathrm{~T}$ low, $\mathrm{HR}=1.9, P=0.444$ ), but showed a significantly longer OS than concordant positive (HER2 positive and H2T high, HR=0.31, $P=0.024$ ). Conversely, the OS curve of discordant high (HER2 negative but $\mathrm{H} 2 \mathrm{~T}$ high, $9 \%$ of all cases) was aligned with concordant positive ( $\mathrm{HR}=0.41, P=0.105)$, but showed a significantly shorter OS than concordant negative ( $H R=41, P<0.0001)$.
\end{abstract}

Conclusions: Quantitative HER2 measurement by HERmark is highly sensitive, accurately quantifies HER2 protein expression and correlates well with routine HER2 testing. When HERmark and local HER2 results were discordant, HERmark more accurately predicted overall survival.

\footnotetext{
* Correspondence: dyardley@tnonc.com

'Sarah Cannon Research Institute, 3322 West End Avenue, Nashville, TN 37203, USA

${ }^{2}$ Tennessee Oncology, PLLC, 250 25th Avenue North, Nashville, TN 37203, USA

Full list of author information is available at the end of the article
} 


\section{Introduction}

Overexpression of human epidermal growth factor receptor 2 (HER2) occurs in approximately 15 to $20 \%$ of primary breast carcinomas and is associated with poor prognosis $[1,2]$. HER2 tumor positivity is also a significant predictive factor for response to HER2-targeted therapies such as trastuzumab (Herceptin ${ }^{\mathrm{Tm}}$ ), pertuzumab $\left(\right.$ Perjeta $\left.^{\mathrm{Tm}}\right)$, lapatinib $\left(\right.$ Tykerb $\left.^{\mathrm{Tm}}\right)$ or trastuzumab emtansine (T-DM1, Kadcyla ${ }^{\mathrm{sm}}$ ) [3-6]. Determination of the HER2 status for all invasive breast cancers at primary diagnosis is now the standard of care $[7,8]$ and can be assessed by various HER2 testing methodologies. While slide-based HER2 assessments on formalin-fixed, paraffin-embedded (FFPE) breast cancer tissues are utilized, routine HER2 testing is subject to significant interlaboratory variation that may result in discrepant results in approximately $20 \%$ of routine HER2 testing in the community [7-10]. Reasons for discordance between laboratory HER2 results are complex and include differences in laboratory proficiencies and performances as well as interpretation of HER2 testing results. This has formed the basis of the expert American Society of Clinical Oncology (ASCO) and the College of American Pathologists (CAP) panel in 2007 to develop guidelines to improve the accuracy of HER2 testing in breast cancer [7]. Recently published reports continue to show lack of concordance for HER2 results between laboratories despite significant emphasis and progress made to standardize routine HER2 testing post publication of the ASCO/CAP guidelines for HER2 testing in 2007 [11-13]. Since then, clarifications and updates to the ASCO/CAP HER2 testing guidelines have been issued, and ASCO and CAP convened to conduct a formal and comprehensive review and revised the guidelines in 2013 [8]. The main objective of HER2 testing remains to accurately determine which patients may benefit from HER2-based targeted therapies.

The HERmark ${ }^{\text {ma }}$ Breast Cancer Assay (HERmark, Monogram Biosciences, South San Francisco, CA, USA) is a validated novel HER2 testing method that provides accurate quantification of HER2 protein in FFPE tissue samples [14,15]. The HERmark assay uses a dualantibody, proximity-based immunoassay approach, the VeraTag $^{\text {Tu }}$ technology (Monogram Biosciences), to make precise and quantitative measurement of total HER2 protein expression $(\mathrm{H} 2 \mathrm{~T})$ with greater sensitivity and specificity than immunohistochemistry (IHC), and provides a continuum of $\mathrm{H} 2 \mathrm{~T}$ values over approximately a 1,000-fold dynamic range in human breast cancers and cell lines [15]. The utilization of capillary electrophoresis for HER2 signal quantification in the HERmark assay yields results that are independent of an observer's subjective interpretation of HER2 signal intensity. A clinical study showed that when breast cancer HER2 expression was measured using the HERmark assay, breast cancer patients with advanced disease whose tumors were HER2 positive by fluorescence in situ hybridization (FISH) but H2T low by HERmark had similar outcomes (as measured by time to progression, following treatment with trastuzumab) as those patients whose cancers were FISH negative and H2T low, thus identifying HER2 FISH-positive tumors with a poorer response to trastuzumab, relative to those tumors which were both HER2 positive by FISH and H2T high by HERmark [16]. These data suggest that HERmark may serve as a novel alternative for quantitative HER2 assessment and provide added value to routine HER2 testing. The aims of the current study were to evaluate the concordance of HER2 results between HERmark and routine HER2 testing methods, and to correlate the results obtained by various HER2 methods with overall survival (OS) of breast cancer patients in a multicenter Collaborative Biomarker Study (CBS) involving 11 study sites.

\section{Methods}

\section{Patient population}

This retrospective, multicenter Collaborative Biomarker Study included women who had tumor tissues from routine surgical excision of invasive breast cancer between January 2000 and May 2005 from the following study sites: Sarah Cannon Research Institute (Nashville, TN, USA)/Tennessee Oncology, PLLC, (Nashville, TN, USA), Dartmouth Hitchcock Medical Center (Lebanon, NH, USA), Texas Oncology Bedford (Bedford, TX, USA), Texas Oncology and Medical City (Dallas, TX, USA), St. Jude Heritage Medical Group (Fullerton, CA, USA), the Center for Cancer and Hematologic Disease (Cherry Hill, NJ, USA), Monroe Medical Associates (Harvey, IL, USA), Swedish American Regional Medical Center (Rockford, IL, USA), the West Clinic (Memphis, TN, USA), Arlington Cancer Center (Arlington, TX, USA), and Wilshire Oncology Medical Group (Rancho Cucamonga, CA, USA). Institutional review board (IRB) approval for the study was conducted by the Western Institutional Review Board (WIRB, Olympia, WA, USA) for our sponsor approval and site IRB approvals. The study was granted a waiver for informed consents and a waiver for authorization under HIPAA, which we specifically requested due to the nature of the study and likelihood that many of the patients whose tumors we studied were now deceased or lost to follow-up. The WIRB determined that this study qualifies for a waiver of consent under 45 CFR 46.116(d). The Board also approved our request for a waiver of authorization for use and disclosure of protected health information (PHI) for the study. Tissue samples and clinical data were anonymized. A total of 232 FFPE breast cancer blocks were collected by the 11 CBS study sites with a median of 22 (range 5 to 27) cases per site. The study was designed to enroll approximately $50 \%$ cases 
with positive HER2 status and 50\% with negative HER2 status as determined by study sites based on local HER2 testing. Freshly cut 5-micron unstained tissue sections were prepared on positively charged glass slides by study sites and sent to Monogram Biosciences for HERmark assay and central HER2 IHC retest in a blinded fashion. Patient-deidentified pathology reports were reviewed and clinicopathological data, including demographics, tumor characteristics, hormone receptor status, treatment (including HER2-targeted therapy received), prior HER2 testing results, and OS data, were collected by study sites, and sent to Monogram Biosciences following the site's receipt of each patient's HERmark result.

\section{The HERmark ${ }^{\mathrm{TM}}$ Breast Cancer Assay}

Total HER2 protein expression $(\mathrm{H} 2 \mathrm{~T})$ was quantified using the HERmark assay as previously described $[14,15,17]$. Briefly, H2T was detected through the proximity-based release of a fluorescent tag conjugated via a cleavable thioether tether, to a monoclonal antibody directed against the cytoplasmic domain of HER2 (Ab8, LabVision, part of Thermo Fisher Scientific, Waltham, MA, USA). The antibody was paired with a biotinylated second antibody directed against the Cterminus of HER2 (Ab15, LabVision, part of Thermo Fisher Scientific). Upon illumination with red light, the photosensitizer molecule linked to the second biotinylated Ab15 liberates singlet oxygen that cleaves any neighboring thioether bonds, thus releasing VeraTag ${ }^{\text {rx }}$ reporters in close proximity. Reporter signal quantified by capillary electrophoresis was normalized to invasive tumor area on the FFPE tissue section. The final value for $\mathrm{H} 2 \mathrm{~T}$ expression (relative fluorescence $(\mathrm{RF}) / \mathrm{mm}^{2}$ tumor) was calculated as (RF concentration) $\times$ (illumination buffer volume)/(tumor area). A total of 194 $(84 \%, 194 / 232)$ collected cases were determined to have adequate invasive breast cancer tissues and yielded valid $\mathrm{H} 2 \mathrm{~T}$ results by HERmark assay. The HERmark assay was performed and H2T results were determined, blinded to local HER2 results and case report form data including clinical outcomes and treatment information. The continuous $\mathrm{H} 2 \mathrm{~T}$ results were categorized as HERmark negative, HERmark equivocal, and HERmark positive with predefined $\mathrm{H} 2 \mathrm{~T}$ analytical cutoff values $\left(<10.5,10.5\right.$ to 17.8 , and $>17.8 \mathrm{RF} / \mathrm{mm}^{2}$, respectively [17]). A pre-defined, published HERmark clinical cutoff $\left(13.8 \mathrm{RF} / \mathrm{mm}^{2}\right.$ [16]) was also used to define $\mathrm{H} 2 \mathrm{~T}$ levels as $\mathrm{H} 2 \mathrm{~T}$ low and $\mathrm{H} 2 \mathrm{~T}$ high in OS analysis.

\section{Central HER2 immunohistochemistry (IHC) retesting}

Central laboratory HER2 IHC retest was performed at the Center for Molecular Biology and Pathology (CMBP, Laboratory Corporation of America, Inc., Research Triangle
Park, NC, USA), using the HercepTest ${ }^{\mathrm{mi}}$ (DAKO, Glostrup, Denmark). A total of 192 (99\%) of the 194 cases that had H2T results yielded valid HER2 IHC results. Central HER2 IHC retest was performed and analyzed blinded to HERmark and local HER2 results. The IHC staining intensity was scored as $0,1+, 2+$, or $3+$ and categorized as HER2 IHC negative, equivocal, or positive according to the ASCO/CAP guideline recommendations for HER2 testing [7].

\section{Local HER2 testing results}

Each study site collected and reported HER2 IHC (defined as local IHC in this study) and/or HER2 FISH (defined as local FISH) results for each case. In addition, each study site reported a final clinical HER2 status (negative, equivocal, or positive, and defined as local HER2 status or investigator-determined HER2 status) based on the combination of all available local HER2 results for each case. Local IHC was reported as $0,1+, 2+$, or $3+$ staining as well as IHC negative, equivocal, or positive as determined by study sites. Local FISH was reported as HER2 amplified or HER2 non-amplified only, and information on HER2/CEP17 ratio was not available for this study. No FISH equivocal case was submitted. Local FISH results were available in 67 (35\%) of the 194 cases that had H2T results.

\section{Statistical analysis}

The agreement between HERmark and HER2 IHC, HER2 FISH- or investigator-determined HER2 status was analyzed according to the number of tumors defined for each category divided by the total number of study patients. Differences in continuous $\mathrm{H} 2 \mathrm{~T}$ values among various categorical HER2 subgroups defined by routine HER2 testing methods were analyzed using the MannWhitney test or the Jonckheere-Terpstra test. Overall concordance that included all HER2 categories as well as positive/negative concordance that excluded equivocal HER2 cases was analyzed in accordance with the ASCO/ CAP HER2 testing guidelines [7]. Kappa statistics were used to evaluate concordance between HERmark and routine HER2 testing methods. OS was defined as the time from initial diagnosis of invasive breast cancer to death or censor. Estimates of survival were based on the Kaplan-Meier (KM) method and statistical significance of differences was evaluated by the log-rank test.

\section{Results}

Patient and tumor characteristics

Patient clinicopathological characteristics are summarized in Table 1. A total of 194 samples that had quantitative HER2 measurements by HERmark were included for analysis. Median age was 51 years (range 27 to 84 years). The majority of tumor samples were of primary 
Table 1 Clinicopathological characteristics of the study population

\begin{tabular}{lll}
\hline Parameter & No. & \% (range) \\
\hline Sample size & 194 & \\
Median length of follow-up (months) & 193 & $67.1(14.8-302.8)$ \\
Median age (years) & & $51(27-84)$ \\
$\quad<40$ & 21 & $11 \%$ \\
$40-49$ & 66 & $34 \%$ \\
$50-59$ & 50 & $26 \%$ \\
$\geq 60$ & 57 & $29 \%$ \\
Menopausal status & & \\
$\quad$ Premenopausal & 75 & $39 \%$ \\
$\quad$ Perimenopausal & 8 & $4 \%$ \\
Postmenopausal & 96 & $49 \%$ \\
$\quad$ Not reported & 15 & $8 \%$
\end{tabular}

Tissue source

Primary breast

Other $^{*}$

Tumor histology

Invasive ductal carcinoma

Invasive lobular carcinoma

Other histologic type

Median tumor size $(\mathrm{cm})$

Not reported

Tumor grade

Grade 1 (well)

Grade 2 (moderate)

Grade 3 (poor)

Not reported

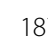

$187 \quad 96 \%$

$7 \quad 4 \%$

tage at initial diagnosis

$\|$

III

IV

Not reported

Nodal status at initial diagnosis

Node positive
Node negative
Not reported
HER2 status (reported)

HER2 status (reported)

Positive

Negative

Equivocal

HER2 IHC (reported)

$$
3+
$$$$
2+
$$

Table 1 Clinicopathological characteristics of the study population (Continued)

\begin{tabular}{lll}
\hline \multicolumn{1}{c}{ IHC 2+/FISH positive } & 2 & $7 \%$ \\
$\quad$ IHC 2+/FISH negative & 18 & $60 \%$ \\
$\quad$ IHC 2+/FISH not reported & 10 & $33 \%$ \\
$1+$ & 32 & $17 \%$ \\
0 & 56 & $29 \%$ \\
HER2 FISH (reported) & & \\
POsitive & 23 & $36 \%$ \\
Negative & 41 & $64 \%$ \\
Hormone receptor (ER/PR) status & & \\
POsitive & 141 & $73 \%$ \\
Negative & 53 & $27 \%$ \\
ER and PR status & & \\
ER (+), PR (+) & 110 & $57 \%$ \\
ER (+), PR (-) & 30 & $15 \%$ \\
ER (-), PR (+) & 1 & $1 \%$ \\
ER (-), PR (-) & 53 & $27 \%$ \\
HER2 targeted therapy & \\
No & & \\
Yes & 174 & $90 \%$ \\
\hline
\end{tabular}

"Other tissue sources: skin, supraclavicular, sentinental lymph node, axillary lymph node, ovary, lung, chest wall; ${ }^{* *}$ other histologic type: 'invasive mammary' (5), breast adenocarcinoma (1), infiltrating mucinous, adenocarcinoma (1), and metastatic carcinoma (1); "trastuzumab- and/or lapatinib-containing therapy. ER, estrogen receptor; FISH, fluorescence in situ hybridization; HER2, human epidermal growth factor receptor 2; IHC, immunohistochemistry; PR, progesterone receptor.

breast cancers (96\%), invasive ductal carcinoma histology (89\%), moderately to poorly differentiated (73\%), stage I or II at initial diagnosis (71\%), and hormone receptor status (estrogen receptor (ER)/progesterone receptor (PR)) positive (73\%). Local HER2 status was reported by the study sites as 57\% HER2 negative, 43\% HER2 positive and 1\% HER2 equivocal. Only $10 \%$ of patients were reported to have received targeted HER2 therapy (trastuzumab and/or lapatinib, Table 1). This retrospective study included women who had tumor tissues from routine surgical excision of invasive breast cancer between January 2000 and May 2005. HER2-targeted therapy was not FDA approved outside of clinical trials for neoadjuvant or early-stage breast cancer therapy during the study period. The median follow-up time was 67.1 months (range 14.8 to 302.8 months).

Comparison of HER2 results and various testing methods The distribution of quantitative total HER2 expression (H2T) was compared with categorical results of routine HER2 tests (Figure 1). Quantitative $\mathrm{H} 2 \mathrm{~T}$ values ranged from 0.3 to $403 \mathrm{RF} / \mathrm{mm}^{2}$ on a continuum for the entire cohort. In comparison with local IHC, the H2T levels ranged from 0.5 to 30.1 (median 4.4) for IHC 0 , from 1.0 


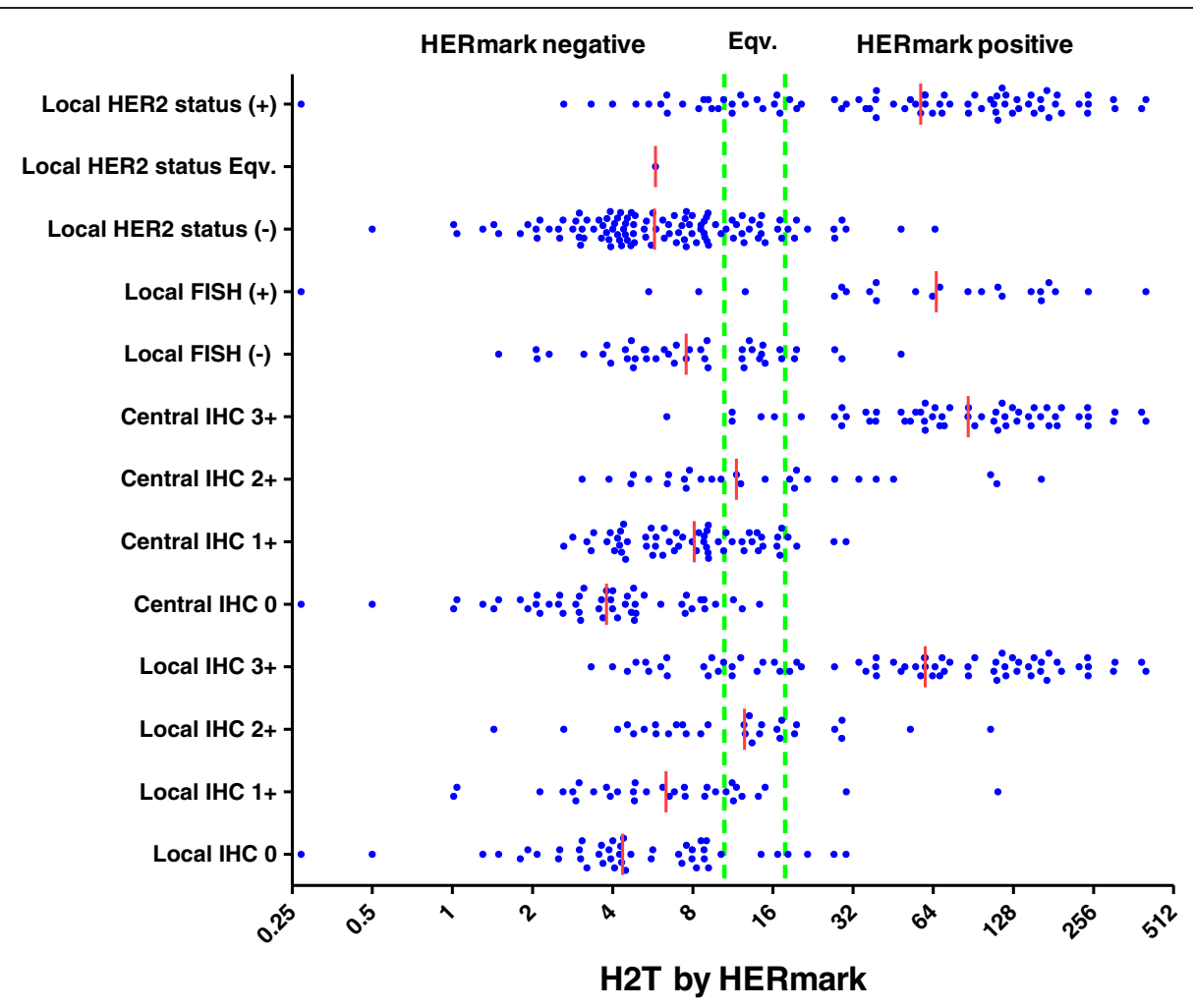

Figure 1 Distribution of quantitative total HER2 expression (H2T) in routine HER2 tests. HERmark equivocal (Eqv.) zone is defined within the two green vertical lines. Short vertical red line indicates the median of a H2T distribution. FISH, fluorescence in situ hybridization; HER2, human epidermal growth factor receptor 2; H2T, quantitative total HER2 expression by HERmark; IHC, immunohistochemistry.

to 112 (median 6.4) for IHC $1+$, from 1.4 to 105 (median 12.5) for IHC 2+, and from 3.3 to 403 (median 59.6) for IHC 3+. Higher H2T levels were significantly associated with stronger IHC staining intensity in the four subgroups by local IHC testing $(P<0.0001$, JonckheereTerpstra test). For central IHC retest cases, the H2T levels ranged from 0.3 to 14.3 (median 3.8) for IHC 0, from 2.6 to 30.1 (median 8.1) for IHC $1+$, from 3.1 to 163 (median 11.7) for IHC 2+, and from 6.4 to 403 (median 86.5) for IHC $3+$. Higher H2T levels were significantly associated with stronger IHC staining intensity in the four central IHC subgroups $(P<0.0001$, JonckheereTerpstra test). In comparison with local FISH, the H2T levels ranged from 1.5 to 48.4 (median 7.6) for FISH negative and from 0.3 to 403 (median 65.7) for FISH positive. In comparing $\mathrm{H} 2 \mathrm{~T}$ levels in FISH-negative versus FISH-positive groups, we observed significantly higher H2T in the FISH-positive group $(P<0.0001$, Mann-Whitney test). For local HER2 status, the H2T levels ranged from 0.5 to 65.0 (median 5.7) for HER2 negative and from 0.3 to 403 (median 57.4) for HER2 positive. Higher H2T levels were significantly associated with local HER2 status positive $(P<0.0001$, Mann-Whitney test). The sole case of local HER2 status equivocal had an $\mathrm{H} 2 \mathrm{~T}$ value of 5.8 and therefore was categorized as HERmark negative.

The comparison between HERmark and routine HER2 tests is presented in Table 2. The overall concordance of all three categories (negative, equivocal, and positive) between HERmark and routine HER2 testing was 68\% (kappa 0.475) for local IHC, 69\% (kappa 0.481) for central IHC, and 73\% (kappa 0.510) for local HER2 status. Analysis of overall concordance between HERmark and local FISH was not performed because there was no FISH equivocal case reported by study sites. When equivocal cases were excluded from all HER2 results, the concordance of positive and negative values between HERmark and routine HER2 testing was 84\% (kappa 0.676) for local IHC, 96\% (kappa 0.914) for central IHC, 85\% (kappa 0.705) for local FISH, and 84\% (kappa 0.682) for local HER2 status.

Kaplan-Meier overall survival analyses by HER2 status as stratified by different HER2 testing methods

The Kaplan-Meier overall survival (OS) analysis was performed on cases that had HER2 testing results and available survival data in 177 cases with local IHC, 188 cases with central IHC, 65 cases with local FISH, 190 cases 
Table 2 Concordance of HERmark with local HER2 IHC, central HER2 IHC, local HER2 FISH, and local clinical HER2 status

\section{Local HER2 IHC Central HER2 IHC Local HER2 FISH}

Local clinical HER2 status

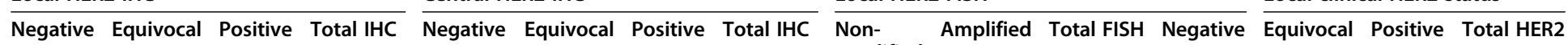
amplified

\begin{tabular}{|c|c|c|c|c|c|c|c|c|c|c|c|c|c|c|c|c|c|c|c|c|c|c|c|c|c|c|c|c|c|c|c|c|}
\hline & \multirow[b]{2}{*}{$\mathrm{N}$} & \multirow[b]{2}{*}{$\%$} & \multirow[b]{2}{*}{$\mathbf{N}$} & \multirow[b]{2}{*}{$\%$} & \multirow[b]{2}{*}{$\mathrm{N}$} & \multirow[b]{2}{*}{$\%$} & \multirow[b]{2}{*}{$\mathrm{N}$} & \multirow[b]{2}{*}{$\%$} & \multirow[b]{2}{*}{$\mathrm{N}$} & \multirow[b]{2}{*}{$\%$} & \multirow[b]{2}{*}{$\mathrm{N}$} & \multirow[b]{2}{*}{$\%$} & \multirow[b]{2}{*}{$\mathrm{N}$} & \multirow[b]{2}{*}{$\%$} & \multirow[b]{2}{*}{$\mathrm{N}$} & \multirow[b]{2}{*}{$\%$} & & \multirow[b]{2}{*}{$\mathrm{N}$} & \multirow[b]{2}{*}{$\%$} & \multirow[b]{2}{*}{$\mathrm{N}$} & \multirow[b]{2}{*}{$\%$} & \multirow[b]{2}{*}{$\mathrm{N}$} & \multirow[b]{2}{*}{$\%$} & \multirow[b]{2}{*}{$\mathrm{N}$} & \multirow[b]{2}{*}{$\%$} & \multirow[b]{2}{*}{$\mathbf{N}$} & \multirow[b]{2}{*}{$\%$} & \multirow[b]{2}{*}{ J } & \multirow[b]{2}{*}{$\%$} \\
\hline & & & & & & & & & & & & & & & & & & & $\mathrm{N}$ & $\%$ & & & & & & & & & & & & \\
\hline HERmark & Negative & & 69 & $71 \%$ & 14 & $14 \%$ & 14 & $14 \%$ & 97 & $51 \%$ & 86 & $86 \%$ & 13 & $13 \%$ & 1 & $1 \%$ & 100 & $52 \%$ & 27 & $90 \%$ & 3 & $10 \%$ & 30 & $45 \%$ & 83 & $83 \%$ & 1 & $1 \%$ & 16 & $16 \%$ & 100 & $52 \%$ \\
\hline HERmark & Equivocal & & 10 & $38 \%$ & 9 & $35 \%$ & 7 & $27 \%$ & 26 & $14 \%$ & 17 & $71 \%$ & 5 & $21 \%$ & 2 & $8 \%$ & 24 & $13 \%$ & 11 & $92 \%$ & 1 & $8 \%$ & 12 & $18 \%$ & 17 & $65 \%$ & 0 & $0 \%$ & 9 & $35 \%$ & 26 & $13 \%$ \\
\hline HERmark & Positive & & 9 & $13 \%$ & 7 & $10 \%$ & 52 & $76 \%$ & 68 & $36 \%$ & 4 & $6 \%$ & 23 & $34 \%$ & 41 & $60 \%$ & 68 & $35 \%$ & 5 & $20 \%$ & 20 & $80 \%$ & 25 & $37 \%$ & 10 & $15 \%$ & 0 & $0 \%$ & 58 & $85 \%$ & 68 & $35 \%$ \\
\hline & & Total & 88 & $46 \%$ & 30 & $16 \%$ & 73 & $38 \%$ & 191 & $100 \%$ & 107 & $56 \%$ & 41 & $21 \%$ & 44 & $23 \%$ & 192 & $100 \%$ & 43 & $64 \%$ & 24 & $36 \%$ & 67 & $100 \%$ & 110 & $57 \%$ & 1 & $1 \%$ & 83 & $43 \%$ & 194 & $100 \%$ \\
\hline
\end{tabular}

Overall

$68 \%,(69+9+52) / 191$

$69 \%,(86+5+41) / 192$

$N A^{\#}$

$73 \%,(83+0+58) / 194$

Kappa (CI 95\%),

0.475 (0.373 to 0.578$)$;

weighted Kappa $=0.545$

0.481 (0.386 to 0.576$)$;

weighted Kappa $=0.631$

0.510 (0.409 to 0.610$)$;

overall

$84 \%,(69+52) /(69+9+14+52)$

$96 \%,(86+41) /(86+4+1+41)$

$85 \%,(27+20) /(27+5+3+20)$

Concordance,
excluding Eqv.

Kappa (CI 95\%),

0.676 (0.550 to 0.797$)$

0.914 (0.841 to 0.988$)$

0.705 (0.516 to 0.893 )

$84 \%,(83+58) /(83+10+16+58)$

excluding Eqv.

"Equivocal (Eqv.) cases from both tests were excluded; ${ }^{\#} \mathrm{NA}$, overall concordance was not calculated for $2 \times 3$ table. Central HER2 IHC retesting was performed retrospectively and central HER2 status was defined per ASCO/CAP guidelines for HER2 testing [7]. The results of local HER2 testing were reported by participating study sites based on local IHC and/or local FISH. Percentages may not add up to $100 \%$ due to rounding. $\mathrm{Cl}$, confidence interval; $\mathrm{FISH}$, fluorescence in situ hybridization; HER2, human epidermal growth factor receptor 2; $\mathrm{HC}$, immunohistochemistry. 
with local HER2 status and 190 cases with HERmark (Figure 2). Local IHC positive (IHC 3+) was associated with a significantly shorter OS compared with local IHC negative $(\mathrm{IHC} 0$ to $1+)$ (Figure 2A: hazard ratio $(\mathrm{HR})=$
2.6; 95\% confidence interval (CI), 1.2 to $5.6 ; P=0.016$ ). OS for local IHC equivocal (IHC 2+) was similar to that of local IHC positive (Figure 2A: HR $=1.0 ; 95 \% \mathrm{CI}, 0.43$ to $2.6 ; P=0.92)$ and showed a trend of shorter OS

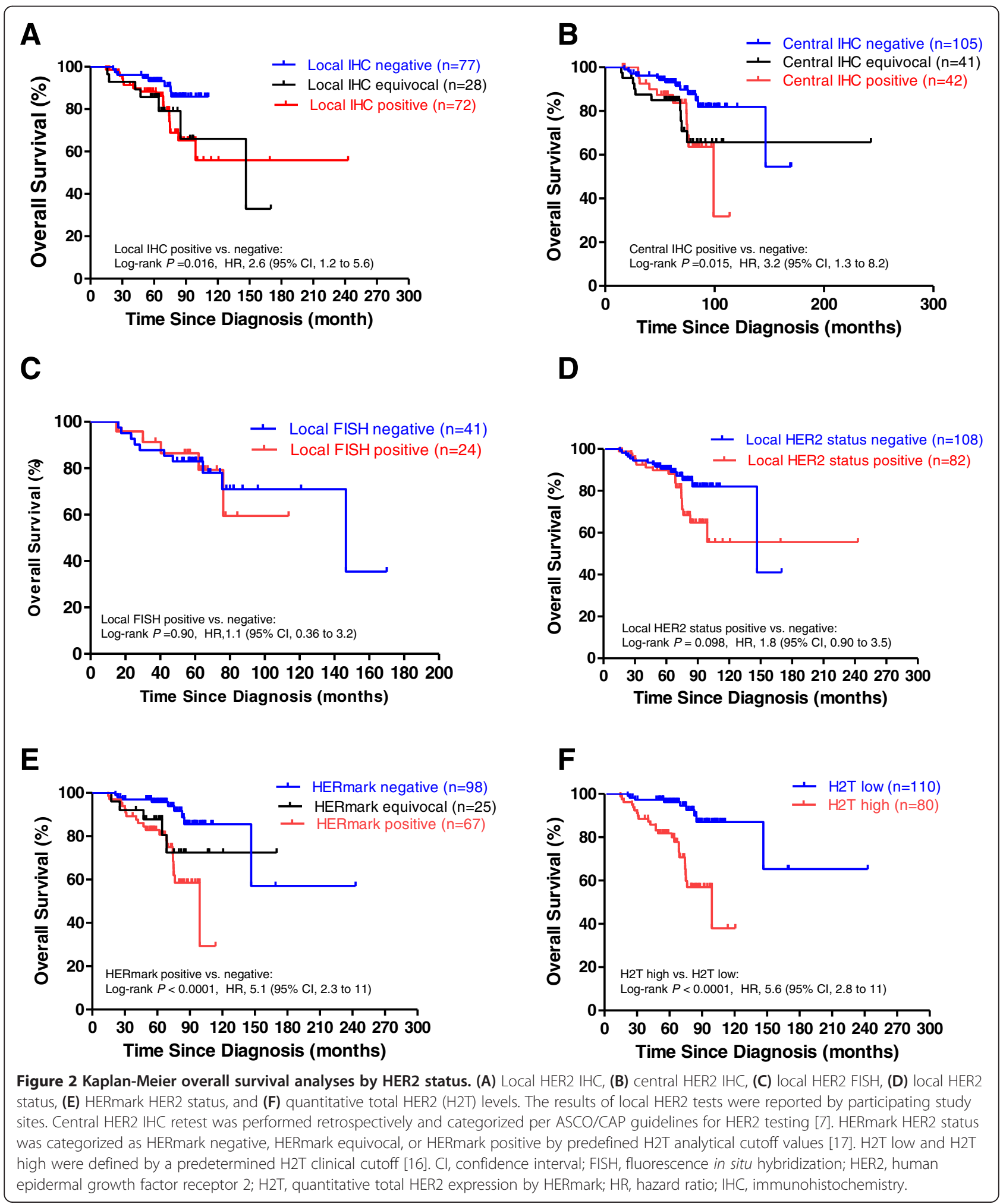


compared with local IHC negative $(\mathrm{HR}=2.7 ; 95 \% \mathrm{CI}$, 0.82 to $9.2 ; P=0.10$ ). Similarly, central IHC positive was associated with a significantly shorter OS compared with central IHC negative (Figure 2B: $\mathrm{HR}=3.2 ; 95 \% \mathrm{CI}, 1.3$ to 8.2; $P=0.015)$. OS in central IHC equivocal was similar to that of central IHC positive (Figure 2B: $H R=0.96$; 95\% CI, 0.41 to $2.3 ; P=0.93$ ) and showed a trend (near statistically significant) of shorter OS compared with the central IHC negative $(\mathrm{HR}=2.5 ; 95 \% \mathrm{CI}, 1.0$ to 6.5 ; $P=0.051$ ). Local FISH positive did not show significant differences in OS compared with local FISH negative (Figure 2C: $\mathrm{HR}=1.1 ; 95 \% \mathrm{CI}, 0.36$ to $3.2 ; P=0.90$ ). Investigator-determined HER2-positive status correlated with a trend of shorter OS compared with HER2negative status (Figure 2D: $\mathrm{HR}=1.8 ; 95 \% \mathrm{CI}, 0.90$ to 3.5 $P=0.098)$. HERmark positive was associated with a significantly shorter OS compared with HERmark negative (Figure 2E: $\mathrm{HR}=5.1 ; 95 \% \mathrm{CI}, 2.3$ to $11 ; P<0.0001$ ). OS of HERmark equivocal was similar to that of HERmark positive (Figure 2E: $\mathrm{HR}=0.62 ; 95 \% \mathrm{CI}, 0.26$ to $1.5 ; P=0.29$ ), and showed a trend of shorter OS compared with HERmark-negative cases $(\mathrm{HR}=3.2,95 \% \mathrm{CI}$,
0.82 to $12 ; P=0.093)$. H2T high $(\mathrm{H} 2 \mathrm{~T}>13.8)$ was associated with a significantly shorter OS compared with H2T low (Figure 2F: $\mathrm{HR}=5.6,95 \% \mathrm{CI}, 2.8$ to $11 ; P<0.0001$ ). When OS analysis for H2T levels was restricted to patients who had not received any HER2-targeted therapy, similar results were observed, showing that H2T high status was associated with a significantly shorter OS compared with H2T low status $(\mathrm{HR}=4.7,95 \% \mathrm{CI}, 1.7$ to $13 ; P=0.003$ ).

The OS correlation between local HER2 status and H2T levels was further explored by plotting the distribution of quantitative H2T levels versus the corresponding local HER2 status and by comparing outcomes in four groups defined by concordant or discordant results using the HERmark H2T clinical cutoff $(\mathrm{H} 2 \mathrm{~T}=13.8$, Figure 3$)$. The distribution of high or low H2T values in local HER2negative and HER2-positive groups overlapped and resulted in four subgroups of H2T distribution (Figure 3A): (1) patients who were HER2 status negative and H2T low (concordant negative, $\mathrm{N}=91,48 \%$ ), (2) patients who were HER2 status positive and H2T high (concordant positive, $\mathrm{N}=63,33 \%$ ), (3) patients who were HER2 status positive
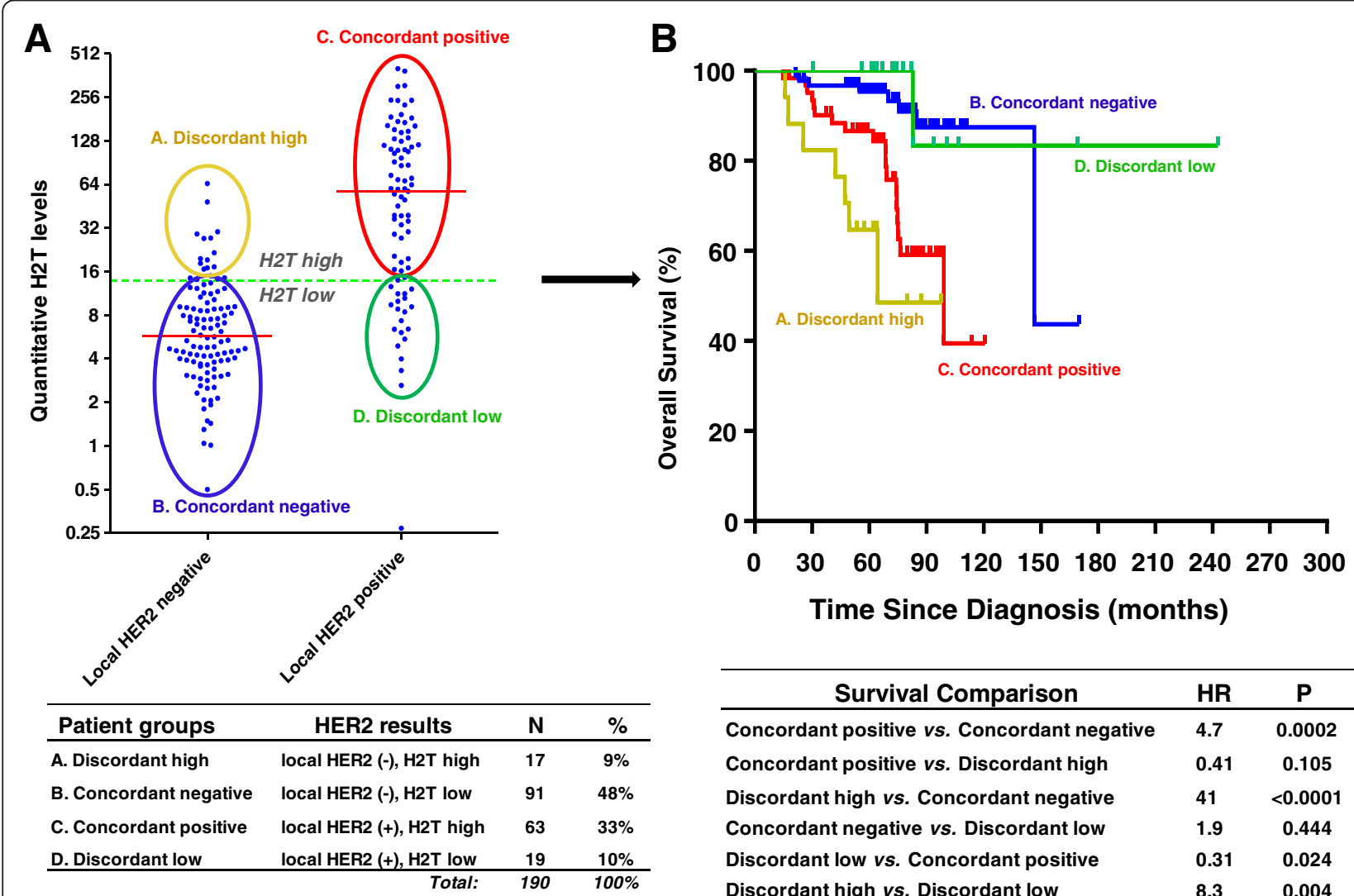

\begin{tabular}{llc}
\hline \multicolumn{1}{c}{ Survival Comparison } & HR & P \\
\hline Concordant positive vs. Concordant negative & 4.7 & 0.0002 \\
Concordant positive vs. Discordant high & 0.41 & 0.105 \\
Discordant high vs. Concordant negative & 41 & $<0.0001$ \\
Concordant negative vs. Discordant low & 1.9 & 0.444 \\
Discordant low vs. Concordant positive & 0.31 & 0.024 \\
Discordant high vs. Discordant low & 8.3 & 0.004 \\
\hline
\end{tabular}

Figure 3 Overall survival and HER2 status as stratified by local HER2 status and H2T levels. (A) H2T distribution by local HER2 status. (B) Kaplan-Meier overall survival analyses for corresponding HER2-H2T concordant and discordant groups. Short horizontal red line in (A) indicates the median of a H2T distribution. HER2, human epidermal growth factor receptor 2; H2T, quantitative total HER2 expression by HERmark; HR, hazard ratio; $P$, the $P$ value of log-rank test. 
but H2T low (discordant low, $\mathrm{N}=19,10 \%$ ) and (4) patients who were HER2 status negative but H2T high (discordant high, $\mathrm{N}=17,9 \%$ ).

Differences in patient clinicopathological characteristics of the concordant and discordant groups are summarized in Table 3. Compared to the concordant negative, cases of the concordant positive were significantly associated with higher tumor grade, higher stage at initial diagnosis, positive nodal status, and negative hormone receptor status. However, no statistically significant differences in the clinicopathological characteristics were found between the two groups that were discordant between local HER2 status and H2T high/low.

Cox proportional hazards multivariate analyses were then conducted to evaluate correlation of HERmark subgroups with OS in the context of clinicopathological variables that showed significant differences between concordant negative and concordant positive (Table 4). Two different sets of cut points were used to define HERmark subgroups for the analyses. In Model A, the 'analytical cut points', that is $<10.5,10.5$ to 17.8 and $>17.8 \mathrm{RF} / \mathrm{mm}^{2}$, were established from HERmark validation study in which $\mathrm{H} 2 \mathrm{~T}$ values were compared with central HER2 IHC/in situ hybridization (ISH) results [17]. The analytical cut points, which define HERmark negative, HERmark equivocal, and HERmark positive, are often used to determine HERmark HER2 status and to compare routine HER2 status by IHC/ISH with HERmark. In Model B, the 'clinical cutoff', that is $13.8 \mathrm{RF} / \mathrm{mm}^{2}$, was established from a HERmark clinical study in which an optimal H2T cut point was established for the purpose for identifying better responders in a clinical cohort of breast cancer patients treated with anti-HER2 therapy [16]. The clinical cut point, which defines H2T high and H2T low, is often used to correlate HERmark results with patient outcome in clinical studies. In both Model A and Model B, HER2 subgroups by HERmark were identified as independent variables that correlate with OS. Patients in HERmark-positive or H2T-high group exhibited significant shorter overall survival compared with patients in HERmark-negative or H2T-low group, respectively. None of the other clinicopathological variables (grade, stage, nodal status, and hormonal status) was identified as an independent variable that significantly correlated with OS.

Next, we performed Kaplan-Meier analysis to evaluate OS for the patients in discordant high group or the discordant low group in comparison with patients in either the concordant-positive group or the concordantnegative group (Figure 3B). The concordant-positive group was associated with a significantly shorter OS compared with the concordant-negative group ( $\mathrm{HR}=$ 4.7, 95\% CI, 2.1 to $11 ; P=0.0002$ ). The discordant-high group generated an OS curve that tracked with the concordant-positive group ( $\mathrm{HR}=0.41,95 \% \mathrm{CI}, 0.14$ to $1.2 ; P=0.105)$, but showed a significantly shorter OS compared with the concordant-negative group $(\mathrm{HR}=41$, 95\% CI, 8.1 to $212 ; P<0.0001)$. The discordant-low group yielded an OS curve that tracked with the

Table 3 Clinicopathologic characteristics of HER2 status-HERmark concordant and discordant groups

\begin{tabular}{|c|c|c|c|c|c|c|c|c|}
\hline \multirow[b]{3}{*}{ Parameter } & \multirow{2}{*}{\multicolumn{2}{|c|}{$\begin{array}{l}\text { Concordant negative } \\
\text { HER2 -, H2T low }\end{array}$}} & \multirow{2}{*}{\multicolumn{2}{|c|}{$\begin{array}{l}\text { Concordant positive } \\
\text { HER2 }+, \text { H2T high }\end{array}$}} & \multirow{2}{*}{\multicolumn{2}{|c|}{$\begin{array}{l}\text { Discordant low } \\
\text { HER2 +, H2T low }\end{array}$}} & \multirow{2}{*}{\multicolumn{2}{|c|}{$\begin{array}{l}\text { Discordant high } \\
\text { HER2 -, H2T high }\end{array}$}} \\
\hline & & & & & & & & \\
\hline & No. & $\%$ & No. & $\%$ & No. & $\%$ & No. & $\%$ \\
\hline \multicolumn{9}{|l|}{ Tumor size $(\mathrm{cm})$} \\
\hline$<2$ & 45 & $51 \%$ & 30 & $49 \%$ & 9 & $47 \%$ & 6 & $43 \%$ \\
\hline$>2$ & 43 & $49 \%$ & 31 & $51 \%$ & 10 & $53 \%$ & 8 & $57 \%$ \\
\hline \multicolumn{9}{|l|}{ Tumor grade } \\
\hline Grade 1 and Grade 2 & 41 & $54 \%$ & 10 & $19 \%^{*}$ & 6 & $40 \%$ & 8 & $62 \%$ \\
\hline Grade 3 & 35 & $46 \%$ & 43 & $81 \%$ & 9 & $60 \%$ & 5 & $38 \%$ \\
\hline \multicolumn{9}{|l|}{ Stage at initial diagnosis } \\
\hline | and || & 73 & $83 \%$ & 40 & $65 \%$ & 14 & $74 \%$ & 8 & $47 \%$ \\
\hline III and IV & 15 & $17 \%$ & 22 & $35 \%{ }^{*}$ & 5 & $26 \%$ & 9 & $53 \%$ \\
\hline \multicolumn{9}{|l|}{ Nodal status at diagnosis } \\
\hline Node negative & 40 & $55 \%$ & 15 & $28 \%$ & 7 & $47 \%$ & 3 & $25 \%$ \\
\hline Node positive & 33 & $45 \%$ & 38 & $72 \%$ & 8 & $53 \%$ & 9 & $75 \%$ \\
\hline \multicolumn{9}{|c|}{ Hormone receptor (ER/PR) status } \\
\hline Negative & 19 & $21 \%$ & 23 & $37 \%{ }^{*}$ & 6 & $32 \%$ & 4 & $24 \%$ \\
\hline Positive & 72 & $79 \%$ & 40 & $63 \%^{*}$ & 13 & $68 \%$ & 13 & $76 \%$ \\
\hline
\end{tabular}

${ }^{*} P<0.05$. Fisher exact test was performed for each parameter between two concordant groups, or, between two discordant groups. ER, estrogen receptor; HER2, human epidermal growth factor receptor 2; H2T, quantitative total HER2 expression by HERmark; PR, progesterone receptor. 
Table 4 Multivariate Cox proportional hazards ratios for association between HER2 measurements by HERmark, selected clinicopathological variables and overall survival

\begin{tabular}{lll}
\hline & \multicolumn{2}{l}{ Overall survival } \\
\cline { 2 - 3 } & HR & P value \\
\hline Model A & 3.1 & 0.034 \\
HERmark positive vs. negative & 2.0 & 0.26 \\
Grade 3 vs. 1 or 2 & 0.93 & 0.89 \\
Stage III or IV vs. I or II & 1.7 & 0.38 \\
Node positive vs. negative & 0.43 & 0.071 \\
Hormone receptor positive vs. negative & & \\
Model B & 3.9 & 0.0061 \\
H2T high vs. Iow & & 0.30 \\
Grade 3 vs. 1 or 2 & 1.7 & 0.84 \\
Stage III or IV vs. I or II & 0.91 & 0.26 \\
Node positive vs. negative & 1.9 & 0.15 \\
Hormone receptor positive vs. negative & 0.54 &
\end{tabular}

"HERmark status based on HERmark analytical cutoffs in Model A: HERmark positive - $\mathrm{H} 2 \mathrm{~T}>17.8$ relative fluorescence (RF) $/ \mathrm{mm}^{2}$; HERmark negative $-\mathrm{H} 2 \mathrm{~T}<10.5$ $\mathrm{RF} / \mathrm{mm}^{2}$. "HER2 levels based on HERmark clinical cutoff in Model B: H2T high - H2T >13.8 RF/mm²; H2T low- H2T $\leq 13.8 \mathrm{RF} / \mathrm{mm}^{2}$. HER2, human epidermal growth factor receptor 2; H2T, quantitative total HER2 expression by HERmark. $\mathrm{HR}$, hazard ratio.

concordant-negative group $(\mathrm{HR}=1.9,95 \% \mathrm{CI}, 0.37$ to 9.9; $P=0.444)$, but showed a significantly longer OS compared with the concordant-positive group $(\mathrm{HR}=$ $0.31,95 \% \mathrm{CI}, 0.11$ to 0.86 ; $\log$-rank $P=0.024)$. In comparing the two discordant groups, the discordant-high group was associated with a significantly shorter OS compared with the discordant-low group ( $\mathrm{HR}=8.3,95 \%$ CI, 1.9 to $35 ; P=0.0042$ ). These results indicate that in the cases with discordance in local HER2 status versus H2T results, the expected overall survival correlates with HERmark results rather than with local HER2 status.

\section{Discussion}

Despite efforts made in the past decade to improve the accuracy and standardization of HER2 testing, discordance in HER2 results between laboratories and testing methodologies persists. The neoadjuvant GeparQuattro trial reported a rather high rate of discordance of $27 \%$ between central and local evaluation of HER2 status [12], which is similar to previous reports of inaccurate local HER2 results in the NSABP B-31 [18] and the NCCTG N9831 studies $[10,19]$. A round-robin study reported a pre-adjudication discordance rate of $8 \%$ for HER2 status (both IHC and FISH) among three expert pathologists from three central laboratories [13], similar to the results from an international HER2 proficiency group study performed among five central laboratories [20]. The main objectives of the current study were to compare HER2 results between a novel HER2 test
(HERmark) and routine HER2 tests (IHC and FISH), and to correlate HER2 results by various HER2 testing methods with overall survival of breast cancer patients from a multicenter Collaborative Biomarker Study.

The quantitative HERmark assay demonstrated a broad continuum (approximately 3 logs) of HER2 expression levels in breast cancer samples in this study. While distribution of $\mathrm{H} 2 \mathrm{~T}$ values correlated significantly with all routine HER2 testing methods, higher concordance was observed between HERmark and central IHC retest as compared to local HER2 tests. This is consistent with previous reports noting higher discordance between local HER2 testing and central HER2 testing relative to discordance among central laboratories $[12,18,19]$. Thus, the HERmark assay may supplement existing routine HER2 testing in the 'real-world' setting as an accurate central HER2 test alternative, particularly for those cases with uncertainty in routine HER2 results, for example, HER2 equivocal cases and HER2 results that show inconsistencies with other clinical and pathological parameters. In addition, the highly sensitive HERmark assay (approximately seven to ten times more sensitive than HER2 IHC $[14,15])$ may serve as a reliable tool to quantify HER2 expression levels in HER2 nonoverexpressing breast cancers where routine HER2 testing may not be optimized to measure lower levels of HER2 protein. Interesting data from the NSABP B31 study suggest that HER2 non-overexpressing breast cancer may benefit from targeted HER2 therapy [21]. For HER2 expression that does not meet the threshold for HER2-positive disease, enrollment into prospective clinical trials is encouraged, such as NCY01275677 (NSABP B47) that aims to address the benefit of adjuvant HER2targeted therapies in tumors with a lower level of HER2 expression. More accurate and quantitative measurement of lower HER2 expression levels may also be helpful for ongoing and planned trials of new anti-HER2 therapies, such as investigational anti-HER2 monoclonal antibodies such as margetuximab and anti-HER2 vaccines targeting HER2 non-overexpressing breast cancer patients [22-24].

We evaluated HER2 status, stratified by routine HER2 testing and HERmark, as a prognostic factor for OS. Most patients in this study did not receive targeted HER2 therapy, and thus, positive HER2 status was expected to correlate with shorter OS. Accordingly, the results showed that HER2 positivity by local IHC, central IHC, or HERmark was significantly associated with a shorter OS compared with HER2 negativity. However, local FISH did not show significant difference in OS between FISH-positive and FISH-negative groups. Possible explanations may include small sample size and selection bias because only a portion of the cases had undergone HER2 FISH testing. Local HER2-positive status, 
which is used routinely in clinical practice to identify patients with HER2-positive tumors for targeted HER2 therapies, showed a trend, but not statistically significant, of shorter OS $(\mathrm{HR}=1.8, P=0.098)$. In contrast, H2T levels, as predefined by a published HERmark clinical cutoff [16], demonstrated a significantly shorter OS in H2T high compared to H2T low $(\mathrm{HR}=5.6, P<0.0001)$.

To further explore the difference in OS between local HER2 status and HERmark H2T results, we performed Kaplan-Meier analysis on four HER2-HERmark subgroups: concordant negative (HER2 negative and H2T low), concordant positive (HER2 positive and H2T high), discordant low (HER2 positive and H2T low), and discordant high (HER2 negative and H2T high), (Figure 3). Each discordant group comprises a significant portion (9 to $10 \%$ ) of the entire cohort. As expected, the concordant-positive group was associated with a significantly shorter OS when compared with the concordantnegative group. Where the results of local HER2 status were discordant with those of HERmark, as in the two discordant groups, the OS curves were significantly aligned with those of HERmark results, but not with the local HER2 status. The results suggest that within the discordant groups, the HERmark results appear to be a better predictor of OS in this clinical cohort. We attempted to explore potential underlying reasons for these differences and compared clinicopathological characteristics of the two discordant groups (Table 3). While concordant positives were significantly associated with higher tumor grade, more advanced stage at initial diagnosis, more positive nodal status, and higher rate of hormone receptor negativity, no significant differences were found in these clinicopathological characteristics between the two discordant groups. In Cox proportional hazards multivariate analyses (Table 4), HER2 expression was identified as the only independent variable that significantly correlates with OS. Although the sample sizes of the discordant groups were relatively small, the difference in OS does not appear to be due to differences in the clinicopathological factors, but likely due to the 'miscall' made by local HER2 status. If these findings are confirmed with further studies, the clinical implications can be significant. Importantly, the results suggest that $10 \%$ of breast cancer patients may be misclassified in routine HER2 testing as false negative, leading to denial of HER2-targeted therapies for patients that could benefit from it. On the other hand, patients misclassified in routine HER2 testing as false positive (approximately 10\%) could lead to the prescribing and administration of costly and potentially toxic ineffective treatment with targeted HER2 therapies. Although the total number of patients selected for anti-HER2 treatment would not change with this scenario, HERmark may offer more accurate selection of breast cancer patients for targeted HER2 therapy in a significant proportion (approximately 20\%) of patients. Obviously, cost and turnaround time implications of additional HER2 tests using a method like HERmark should be further evaluated. Recently, a study of cost-effectiveness for expanded HER2 reflex testing was reported [25]. The analysis showed that retesting patients who are $\mathrm{IHC} 0, \mathrm{IHC1+}$, or FISH-negative is projected to be a cost-effective clinical strategy. The expanded reflex testing allows for a second opportunity to measure HER2 status accurately, correcting both handling errors and testing inconsistency. Similarly, we believe that selective HER2 retest by HERmark for patients who had discordant or inconclusive HER2 status by IHC/ISH would also be a cost-effective clinical strategy, which warrants further investigation.

The strengths of this study include the multicenter setting which represents 'real-world' routine HER2 testing in the field and thus, is relevant to clinical practice. Evaluation of various HER2 results, including those of routine local and central HER2 tests coupled with the novel HERmark HER2 quantification, offers a comprehensive comparison among various HER2 testing methods. Despite the heterogeneous therapies in the clinical patient cohort from various study sites, the majority of patients did not receive HER2-targeted therapy and thus OS is a relatively reliable clinical outcome in such a setting. The study has several limitations. The retrospective nature of this biomarker study carries inherent heterogeneities that exist not only in the tumor specimens evaluated but also in patient selection, HER2 testing methods, and treatments. The study was designed to include approximately 50\% HER2-positive and 50\% HER2-negative cases for effective comparison of HER2 results by various testing methods, and thus does not represent HER2 status in a general population of breast cancer patients in which the expected rate of HER2 positivity is approximately $15 \%$. The number of cases in each of the two discordant groups is relatively small. Thus, the issues with relatively small groups in a relatively skewed patient population should be addressed in future studies. Since most patients in this study did not receive targeted HER2 therapy, further studies are also needed to investigate anti-HER2 response in patients of the discordant groups.

\section{Conclusions}

The novel HERmark assay offers highly sensitive and accurate quantification of HER2 protein expression that has demonstrated excellent concordance with central HER2 testing and better correlation as a prognostic factor in OS as compared with 'real-world' local HER2 status in a multicenter clinical cohort of breast cancer patients. The HERmark assay may reclassify $10 \%$ of false-negative patients by conventional tests as truly positive and $10 \%$ who are currently testing false positive 
by conventional tests as negative. Thus, a resultant change in therapy for $20 \%$ of patients may improve the outcomes for both HER2-negative and -positive cohorts. Further clinical studies are warranted to confirm these findings within well-controlled clinical cohorts and clinical trials.

\section{Abbreviations}

Cl: confidence interval; ER: estrogen receptor; FFPE: formalin-fixed, paraffin-embedded; FISH: fluorescence in situ hybridization; HER2: human epidermal growth factor receptor 2; H2T: quantitative total HER2 expression by HERmark; HR: hazard ratio; IHC: immunohistochemistry; ISH: in situ hybridization; OS: overall survival; PR: progesterone receptor; RF: relative fluorescence.

\section{Competing interests}

$\mathrm{WH}, \mathrm{YL}$ and $\mathrm{JS}$ are employees of Monogram Biosciences, and $\mathrm{SA}$ and $\mathrm{MB}$ are employees of Monogram's parent company, Laboratory Corporation of America. RP, ACP, JW, MH and MPB are former employees of Monogram Biosciences. There were no competing interests for all other authors.

\section{Authors' contributions}

All authors have made substantial contributions to conception and design, or acquisition of data, or analysis and interpretation of data, and have been involved in drafting the manuscript or revising it critically for important intellectual content. Specifically, DAY, PAK, WH, RP, MPB, JW and LB were involved in the conception and design of the study. WH, JW, LB, SA drafted the manuscript and WH, JW, LB, SA, DAY, PAK, LK, MS, WEL, SZ, AS, HE, LSS, JWA, YL, ACP, JS, MPB, RP and MB revised it for intellectual content. WH, JS, $\mathrm{MH}$, and $\mathrm{ACP}$ performed the statistical analysis. MB carried out the central lab HER2 immunohistochemistry assays. WH was the study pathologist, and carried out the pathology review of all tumor specimens for HERmark testing. $Y L$ was responsible for clinical data management and sample handling. DAY, PAK, LK, MS, WEL, SZ, AS, HE, LSS, JWA, and LB were the study investigators who provided the study materials/patients. All authors have read and approved the final manuscript.

\section{Acknowledgements}

The authors wish to thank the research coordinators and cooperating pathologists of the 11 CBS study sites for data collection and tissue sample preparation. We would also like to thank the clinical laboratory at the Center for Molecular Biology and Pathology (CMBP, Research Triangle Park, NC) for performing central HER2 IHC retesting, and the Oncology Clinical Reference Laboratory at Monogram Biosciences (South San Francisco, CA) for their assistance in generating the HERmark assay data, and Cynthia Sedik and Gabriella Arroyo for assistance with data entry and management. We would like to acknowledge the contribution of former Monogram Biosciences employees: Thomas Sherwood (currently at Cepheid, Sunnyvale, CA), Smitha Sivaraman (currently at Incyte, Delaware, DE), and Soumi Gupta (currently at Janssen Services, LLC, Titusville, NJ).

\section{Author details}

'Sarah Cannon Research Institute, 3322 West End Avenue, Nashville, TN 37203, USA. ${ }^{2}$ Tennessee Oncology, PLLC, 250 25th Avenue North, Nashville, TN 37203, USA. ${ }^{3}$ Dartmouth Hitchcock Medical Center, 1 Medical Center Drive, Lebanon, NH 03766, USA. ${ }^{4}$ Monogram Biosciences, Inc., 345 Oyster Point Boulevard, South San Francisco, CA 37203, USA. ${ }^{5}$ Texas Oncology Bedford, 1615 Hospital Parkway, Bedford, TX 76022, USA. ${ }^{6}$ Texas Oncology and Medical City, 7777 Forest Lane, Dallas, TX 75230, USA. ${ }^{7}$ St. Jude Heritage Medical Group, 2720 Harbor Boulevard, Fullerton, CA 92835, USA. ${ }^{8}$ The Center for Cancer and Hematologic Disease, 1930 New Jersey 70 (East), Cherry Hill, NJ 08003, USA. ${ }^{9}$ Monroe Medical Associates, 71 Ald Taylor Way, Harvey, IL 60426, USA. ${ }^{10}$ Swedish American Regional Medical Center, 1401 East State Street, Rockford, IL 61104, USA. ${ }^{11}$ The West Clinic, 100 N Humphreys Boulevard, Memphis, TN 38120, USA. ${ }^{12}$ Arlington Cancer Center, 906 West Randol Mill Road, Arlington, TX 76012, USA. ${ }^{13}$ Center for Molecular Biology and Pathology, Laboratory Corporation of America, Inc, Research Triangle Park, NC 27709, USA. ${ }^{14}$ Wilshire Oncology Medical Group, 8283 Grove Avenue, Rancho Cucamonga, CA 91730, USA. ${ }^{15}$ Present address: Institut de Pharmacologie Moléculaire et Cellulaire-IPMC, Sophia Antipolis, 660 Route des Lucioles, 06560 Valbonne, France. ${ }^{16}$ Present address: HealthTell, 3130 Crow Canyon Place, San Ramon, CA
94583, USA. ${ }^{17}$ Present address: Quest Diagnostics, 33608 Ortega Highway, San Juan Capistrano, CA 92675, USA. ${ }^{18}$ Present address: Cepheid, 904 East Caribbean Drive, Sunnyvale, CA 94089, USA. ${ }^{19}$ Present address: City of Hope, 1500 East Duarte Road, Rancho Cucamonga, CA 91010, USA.

Received: 14 October 2014 Accepted: 27 February 2015 Published online: 18 March 2015

\section{References}

1. Slamon DJ, Godolphin W, Jones LA, Holt JA, Wong SG, Keith DE, et al. Studies of the HER2/neu proto-oncogene in human breast and ovarian cancer. Science. 1989;244:707-12.

2. Yarden Y, Sliwkowski MX. Untangling the ErbB signaling network. Nat Rev Mol Cell Biol. 2001;2:127-37.

3. Slamon DJ, Clark GM, Wong SG, Levin WJ, Ullrich A, McGuire WL. Human breast cancer: correlation of relapse and survival with amplification of the HER-2/neu oncogene. Science. 1987;235:177-82.

4. Baselga J, Gelmon KA, Verma S, Wardley A, Conte P, Miles D, et al. Phase II trial of pertuzumab and trastuzumab in patients with human epidermal growth factor receptor 2-positive metastatic breast cancer that progressed during prior trastuzumab therapy. J Clin Oncol. 2010;28:1138-44.

5. Geyer CE, Forster J, Lindquist D, Chan S, Romieu CG, Pienkowski T, et al. Lapatinib plus capecitabine for HER2-positive advanced breast cancer. N Engl J Med. 2006;355:2733-43.

6. Burris III HA, Rugo HS, Vukelja SJ, Vogel CL, Borson RA, Limentani S, et al. Phase II study of the antibody drug conjugate trastuzumab-DM1 for the treatment of human epidermal growth factor receptor 2 (HER2)-positive breast cancer after prior HER2-directed therapy. J Clin Oncol. 2011;29:398-405.

7. Wolff AC, Hammond ME, Schwartz JN, Hagerty KL, Allred DC, Cote RJ, et al. American Society of Clinical Oncology/College of American Pathologists guideline recommendations for human epidermal growth factor receptor 2 testing in breast cancer. J Clin Oncol. 2007;25:118-45.

8. Wolff AC, Hammond ME, Hicks DG, Dowsett M, McShane LM, Allison KH, et al. Recommendations for human epidermal growth factor receptor 2 testing in breast cancer: American Society of Clinical Oncology/College of American Pathologists clinical practice guideline update. J Clin Oncol. 2013;31:3997-4013.

9. Perez EA, Roche PC, Jenkins RB, Reynolds CA, Halling KC, Ingle JN, et al. HER2 testing in patients with breast cancer: poor correlation between weak positivity by immunohistochemistry and gene amplification by fluorescence in situ hybridization. Mayo Clin Proc. 2002;77:148-54.

10. Roche PC, Suman VJ, Jenkins RB, Davidson NE, Martino S, Kaufman PA, et al. Concordance between local and central laboratory HER2 testing in the Breast Intergroup Trial N9831. J Natl Cancer Inst. 2002;94:855-7.

11. Mollerup J, Henriksen U, Müller S, Schønau A. Dual color chromogenic in situ hybridization for determination of HER2 status in breast cancer: a large comparative study to current state of the art fluorescence in situ hybridization. BMC Clin Pathol. 2012;12:3.

12. Denkert C, Huober J, Loibl S, Prinzler J, Kronenwett R, Darb-Esfahani S, et al. HER2 and ESR1 mRNA expression levels and response to neoadjuvant trastuzumab plus chemotherapy in patients with primary breast cancer. Breast Cancer Res. 2013;15:R11.

13. Perez EA, Press MF, Dueck AC, Jenkins RB, Kim C, Chen B, et al. Immunohistochemistry and fluorescence in situ hybridization assessment of HER2 in clinical trials of adjuvant therapy for breast cancer (NCCTG N9831, BCIRG 006, and BCIRG 005). Breast Cancer Res Treat. 2013;138:99-108.

14. Shi $Y$, Huang $W$, Tan $Y$, Jin $X$, Dua R, Penuel E, et al. A novel proximity assay for the detection of proteins and protein complexes: quantitation of HER1 and HER2 total protein expression and homodimerization in formalin-fixed, paraffin-embedded cell lines and breast cancer tissue. Diagn Mol Pathol. 2009;18:11-21.

15. Larson JS, Goodman L, Tan Y, Defazio-Eli L, Paquet AC, Cook JW, et al. Analytical validation of a highly quantitative, sensitive, accurate, and reproducible assay (HERmark) for the measurement of HER2 total protein and HER2 homodimers in FFPE breast cancer tumor specimens. Patholog Res Int. 2010;28:814176.

16. Lipton A, Köstler WJ, Leitzel K, Ali SM, Sperinde J, Weidler J, et al. Quantitative HER2 protein levels predict outcome in fluorescence in situ hybridization-positive patients with metastatic breast cancer treated with trastuzumab. Cancer. 2010;116:5168-78. 
17. Huang W, Reinholz M, Weidler J, Yolanda L, Paquet A, Whitcomb J, et al. Comparison of central HER2 testing with quantitative total HER2 expression and HER2 homodimer measurements using a novel proximity-based assay. Am J Clin Pathol. 2010;134:303-11.

18. Paik S, Bryant J, Tan-Chiu E, Romond E, Hiller W, Park K, et al. Real-world performance of HER2 testing-national surgical adjuvant breast and bowel project experience. J Natl Cancer Inst. 2002;94:852-4.

19. Perez EA, Suman VJ, Davidson NE, Martino S, Kaufman PA, Lingle WL, et al. HER2 testing by local central, and reference laboratories in specimens from the North Central Cancer Treatment Group N9831 intergroup adjuvant trial. J Clin Oncol. 2006;24:3032-8.

20. Dowsett M, Hanna WM, Kockx M, Penault-Llorca F, Rüschoff J, Gutjahr T, et al. Standardization of HER2 testing: results of an international proficiency-testing ring study. Mod Pathol. 2007;20:584-91.

21. Paik S, Kim C, Wolmark N. HER2 status and benefit from adjuvant trastuzumab in breast cancer. N Engl J Med. 2008;358:1409-11.

22. Sears AK, Perez SA, Clifton GT, Benavides LC, Gates JD, Clive KS, et al. AE37: a novel T-cell-eliciting vaccine for breast cancer. Expert Opin Biol Ther. 2011;11:1543-50.

23. Mittendorf EA, Clifton GT, Holmes JP, Clive KS, Patil R, Benavides LC, et al. Clinical trial results of the HER-2/neu (E75) vaccine to prevent breast cancer recurrence in high-risk patients: from US military cancer institute clinical trials group study I-01 and I-02. Cancer. 2012;1 18:2594-602.

24. Pegram MD, Tan-Chiu, Miller K, Rugo HS, Yardley DA, Liv S, et al. A single-arm, open-label, phase 2 study of MGAH22 (margetuximab) [fc-optimized chimeric anti-HER2 monoclonal antibody (mAb)] in patients with relapsed or refractory advanced breast cancer whose tumors express HER2 at the $2+$ level by immunohistochemistry and lack evidence of HER2 gene amplification by FISH. J Clin Oncol. 32:5s, 2014; (suppl; abstr TPS671^)

25. Garrison Jr LP, Lalla D, Brammer M, Babigumira JB, Wang B, Perez EA Assessing the potential cost-effectiveness of retesting $\mathrm{IHC}, \mathrm{IHC1+}$, or FISH-negative early stage breast cancer patients for HER2 status. Cancer. 2013;119:3113-22

\section{Submit your next manuscript to BioMed Central and take full advantage of:}

- Convenient online submission

- Thorough peer review

- No space constraints or color figure charges

- Immediate publication on acceptance

- Inclusion in PubMed, CAS, Scopus and Google Scholar

- Research which is freely available for redistribution 